\title{
"La inclusión de los padres en el cuadro de la situación analítica". Familia, disciplinas psi y valores en la Argentina de los cincuenta*
}

"The Inclusion of Parents in the Picture of the Analytic Situation". Family Psi Disciplines and Values in the Fifties Argentina

Recibido: diciembre 2 de 2013 | Revisado: enero 20 de 2014 | Aceptado: julio 17 de 2014

FLORENCIA ADRIANA MACCHIOLI**

Universidad de Buenos Aires, Argentina

doi:10.11144/Javeriana.upsy13-5.ipcs

Para citar este artículo: Macchioli, F. A. (2014). "La inclusión de los padres en el cuadro de la situación analítica". Familia, disciplinas psi y valores en la Argentina de los cincuenta. Universitas Psychologica, 13(5), 1881-1891. http://dx.doi.org/10.11144/ Javeriana.upsy13-5.ipcs

Artículo de investigación en el marco del Proyecto de Investigación UBACyT "Conocimiento, prácticas y valores en la historia de la psicología y del psicoanálisis en la Argentina”, 2011-2014, código 20020100100627. Agradezco especialmente los comentarios de Ana María Talak, Luciano García e Isabella Cosse.

** Correo electrónico: famacchioli@yahoo.com.ar

\section{RES U MEN}

El trabajo explora la delimitación de una zona de problemas en torno al niño y la familia, entendidos como objeto de intervención psicológica, durante la década de los años cincuenta, en la Argentina. A partir de un caso clínico de Arminda Aberastury publicado en 1957 en la Revista de Psicoanálisis, se abordan varias dimensiones de análisis, desde un abordaje historiográfico que plantea un cruce entre la historia de las disciplinas psi y los valores. Se hace hincapié en el modo en que se vinculan los valores atribuidos al individuo (especialmente al niño) y a la familia, así como los criterios de saludenfermedad, los roles de género masculino-femenino y las implicancias de estos valores en los saberes y prácticas del período.

Palabras clave

psicoanálisis; psiquiatría; historia; prácticas

\section{A B S T R A C T}

This is an exploration of the boundaries of problem areas in the study of the child and the family seen as the object for psychological intervention in Argentina in the 1950ies. Based on a clinical history published by Arminda Aberastury in 1957 in Revista de Psicoanálisis, several analytical approaches are discussed from a historiographic perspective in the light of the history of psy-disciplines and a theory of values. The emphasis is on the connecting thread between the values attached to the individual (the child) and the family, as well as health/sickness criteria, gender roles, and the significance of those in the knowledge and understanding informing the clinical practices at the time.

Keywords

psychoanalysis; psychiatry; history; practices 
Alrededor de la década del cuarenta, comienza a practicarse en la Argentina el psicoanálisis de niños. Este hecho estaba propiciado por la temprana recepción del freudismo en la Argentina, a principios del siglo XX (Vezzetti, 1985, 1996a, 1996b) y, algunas décadas después, por la fundación en 1942 de la Asociación Psicoanalítica Argentina (APA). Y así como los favorables vientos de cambio traían el psicoanálisis de niños europeo a las latitudes australes, este viajaba a su vez dentro del continente y nuevamente a Europa en un recorrido que lo nutría y transformaba (Bardassi, 2009; Dagfal, 2009; Macchioli, 2012; Oliveira, 2002).

En el presente trabajo, se seguirán los desarrollos de la historia intelectual, en especial sus líneas de investigación sobre la historia de las prácticas y circulación de saberes (Dafermos, Marvakis, Mentinis, Painter, \& Triliva, 2013; Dotti, Blanco, Plotkin, García, \& Vezzetti, 2008; Pulido, 2007; Tarcus, 2007; Vera-Villarroel, López-López, Lillo, \& Silva, 2011; Vezzetti, 2007a). Pero aquí se añadirán a los abordajes histórico-críticos de las disciplinas psi la articulación con los valores ${ }^{11}$. Dicha postura sostiene que estos intervienen en modo directo en la selección de evidencia empírica con la que se construyen las teorías (Dorlin, 2009; Prilleltensky, 1997; Talak, 2009).

El siglo XX fue denominado por algunos autores como el "siglo del niño", frase que concentró cierta utopía de transformación en la que el niño simbolizaba tanto el motor como el destino del cambio social. Y así como durante fines del siglo XIX y la primera mitad del siglo XX el sistema educativo escolar y ciertas interpretaciones pedagógicas de la naturaleza del niño eran las que trazaban las pautas sobre la concepción de la infancia; a partir de los sesenta, será el psicoanálisis de niños el que pretenderá ubicarse en una posición estratégica respecto a la construcción de una nueva verdad sobre la infancia (Carli, 2009, p. 14). Aunque previamente al psicoanálisis, ya la pediatría, la psicología y la

1 Se entenderá aquí por "valores" a las pautas morales, éticas y procedimentales con las que se evalúan a los usuarios, a los fenómenos psicológicos, a las técnicas y a sus efectos de manera explícita o implícita (Benito \& García, 2010, p. 62). psiquiatría infantil comenzaron a trazar los límites entre lo normal y lo patológico en la infancia y, desde allí, el modo en que fueron concebidos los conceptos de salud y enfermedad (Borinsky, 2009).

En esta dirección, Talak (en prensa) sostiene que el problema del niño, planteado desde fines del siglo XIX en la Argentina, parte de los problemas urbanos y de la cuestión social. En particular, la criminalidad infantil poseía una importante repercusión en la opinión pública y justificaba, en gran medida, la necesidad tanto de la intervención del Estado a partir de políticas sociales como desde el ámbito familiar privado. Diversas problemáticas, como la indisciplina escolar o la delincuencia infantil o juvenil, constituyeron un problema que demandó simultáneamente saberes teóricos e intervenciones profesionales y políticas que estimularon la producción de nuevos saberes interdisciplinarios, inseparables de las ideas de época y de las políticas públicas vigentes. En este escenario, la psicología ofreció un marco general que favoreció lecturas y explicaciones del desarrollo psicológico que asumieron tanto valoraciones de época como de ciertos grupos sociales, propiciando "una doble direccionalidad en las relaciones entre conocimientos, prácticas y valoraciones de la psicología y de los grupos sociales a los que pertenecían los autores que investigaban estos temas" (Talak, en prensa, p. 31). En especial, el examen clínico de sujetos individuales que presentaban trastornos o anomalías seguía el modelo de la psicopatología y la clínica francesas, a las que se sumó, a partir de la década de 1940, el psicoanálisis de corte inglés. A nivel local, se articuló con la clínica psiquiátrica y permitió a su vez a los mismos psiquiatras ampliar su intervención a patologías menos graves que dieron lugar a los tratamientos ambulatorios, a la psicoterapia y al psicoanálisis. Estos abordajes se centraron primero en los adultos y poco después en los niños, particularmente en los casos de enuresis, tartamudez, discapacidades sensoriales, retraso mental y posteriormente a los problemas de delincuencia, prostitución infantil y al "niño débil". Estos desarrollos fueron "un componente fundamental de la grilla interpretativa psicologizada desde la cual se leían y abordaban otros problemas, interpretados 
"LA INCLUSIÓN DE LOS PADRES EN EL CUADRO DE LA SITUACIÓN ANALÍTICA".

FAMILIA, DISCIPLINAS PSI Y VALORES EN LA ARGENTINA DE LOS CINCUENTA

en términos de anormalidad y patología" (Talak, en prensa, p. 35).

Estos lineamientos pueden observarse en el caso clínico que aquí se abordará, a la vez que deberá tenerse en cuenta que se publica en 1957, año que marca un punto de viraje en la conformación del campo psi local, a partir de la fundación del Instituto Nacional de Salud Mental, la inauguración de los servicios de psicopatología en los hospitales generales, la creación de la carrera de Psicología en la Universidad de Buenos Aires entre otras casas de estudio y diversas experiencias que iniciaban en el segundo lustro de los cincuenta, como la Escuela para Padres o las terapias de grupo, entre otras, marcando el pasaje de la higiene mental a la salud mental (Borinsky, 2009; Carpintero \& Vainer, 2004, 2005; Dagfal, 2009; Macchioli, 2013; Plotkin, 2003; Vezzetti, 2007b).

A partir de estas premisas, el presente trabajo explorará el modo en que se delimitó una zona de problemas en la Argentina en torno al niño, pero también a la familia, entendidos ambos como objetos de intervención psicológica. Se tendrá en cuenta el modo en que se vinculan los valores atribuidos al individuo (especialmente al niño) y a la familia, así como los criterios de salud-enfermedad, los roles de género masculino y femenino y las implicancias de estos valores en los saberes y prácticas de la época.

Con estos objetivos, se analizará aquí un caso clínico de Arminda Aberastury de Pichon-Rivière, titulado "La inclusión de los padres en el cuadro de la situación analítica y el manejo de esta situación a través de la interpretación", publicado en 1957, en la Revista de Psicoanálisis editada por la APA. Este artículo, al condensar diversos tópicos, permite examinar de diversos modos los cruces entre psicoanálisis e infancia. Por una parte, explorar el modo en que se constituyó el psicoanálisis de niños en la Argentina entre las décadas de 1940 y 1950, en el escenario descripto donde se cruzan la pediatría, la psiquiatría, la criminología, la psicología y la pedagogía con los saberes y prácticas psicoanalíticos. Por otra parte, este caso en particular permite focalizar las dificultades de establecer delimitaciones entre el niño y la familia, dibujando las fronteras difusas que darán lugar más adelante a la demarcación de un campo específico para las intervenciones familiares (Macchioli, 2010). Asimismo, permite estudiar el modo en que se inscriben estos saberes y prácticas no solamente en la coyuntura psi del período, sino también con la delimitación de nuevos objetos de intervención, principalmente el grupo y la familia. En este sentido, el caso clínico se encuentra en los umbrales de lo que en los años sesenta será una transición que dará lugar, entre otras cosas, a la redefinición radical del lugar de la mujer en la sociedad, en la familia y en el ámbito laboral (Cosse, 2010; Nari, 2004; Torrado, 2004; Wainerman, 2005). Finalmente, se considerarán ciertos valores disciplinares presentes en las prácticas de la época, entre ellos los relativos a los criterios se saludenfermedad, a los roles masculinos y femeninos, así como también a las distinciones entre paciente, niño, padres, familia y grupo.

Pero antes, una semblanza de Arminda Aberastury (1910-1972). En 1929, se graduó como maestra en la Escuela Normal №6 de la Ciudad de Buenos Aires y en 1937 se inscribió en la recién inaugurada carrera de Pedagogía en la Facultad de Filosofía y Letras de la Universidad de Buenos Aires. En esta etapa de formación pedagógica, Aberatury "combinó lecturas de filosofía, pedagogía y psicología", etapa que "coexistió con su primer acercamiento al psicoanálisis que se produce durante los años 30" (Carli, 2009, p. 15). En 1937, trabaja en salas de lactantes y poco después iniciarían sus primeros tratamientos psicoanalíticos infantiles al fondo del pabellón psiquiátrico que dirigía Pichon-Rivière, su marido, en el Consultorio de la Liga de Higiene Mental del Hospicio de las Mercedes ${ }^{2}$. Una de

2 A través de Federico Aberastury, uno de sus hermanos, conoció a Enrique Pichon-Rivière, su futuro esposo. Esta pareja cumplió un importante rol en el proceso de institucionalización del psicoanálisis en la Argentina, a partir de la fundación de la Asociación Psicoanalítica Argentina en 1942. La unión duró de 1937 a 1956, período en el que tuvieron tres hijos. A la edad de 62 años Aberastury se quitó la vida, ingiriendo una sobredosis de medicamentos, muerte que fue mitificada por la historia oficial como efecto de una enfermedad deformante en la piel. A lo largo de su recorrido profesional, fue una de las exponentes principales en la recepción, formación y práctica del psicoanálisis infantil en la Argentina y su difusión en Uruguay y Brasil. Produjo la primera traducción al castellano de las obras de Melanie Klein y trabajó interdisciplinariamente con odontopediatras, grupos de madres desde 1961, adolescencia (tópico poco explorado hasta ese 
las tardes que pasaba a buscarlo por la institución (Puget, 2011), Aberastury conoció casualmente en la sala de espera a una niña de 8 años que esperaba a su madre, tratada por Pichon-Rivière. Comenzó a tratar a la niña siguiendo la técnica psicoanalítica descrita por Anna Freud (Aberastury, 1962). Este caso, ubicado como el primer tratamiento psicoanalítico infantil en la Argentina, marca el comienzo de una clínica de niños que intervino radicalmente en la formación de las posteriores generaciones de psicoanalistas.

A partir de la lectura de Psicoanálisis de niños de Klein, alrededor de 1945, Aberastury le escribe una carta a la autora donde manifiesta algunas dificultades respecto a la relación con los padres, al manejo del material de juego y al pago de honorarios. Por otra parte, le anuncia que había iniciado la traducción de su libro al castellano y solicita la publicación de un artículo en el que había citado a Anna Freud y a Melanie Klein en el International Journal of Psycho-Analysis. Klein responde a los interrogantes planteados por Aberastury, pero agrega que su artículo no cumple los estándares internacionales de publicación. Aunque lo más probable es que, como ella no conocía las controversias entre Melanie Klein y Anna Freud -que prácticamente habían escindido la Sociedad Británica- ese haya sido el principal motivo para negarse a publicar el artículo. Esta primera respuesta de Klein la recibió el 27 de abril de 1945, y a partir de allí iniciaron un intercambio epistolar que duró hasta 1957, cuando se publica el caso que aquí se estudia (Borinsky, 2009; Geissman \& Geissman, 2002).

Entre las décadas de 1940 y 1960, a partir de la recepción de aportes ingleses y franceses predominantemente, se produce una mixtura singular en los desarrollos del psicoanálisis argentino. Dagfal (2009) analizó la dinámica del triángulo Buenos Aires-París-Londres, observando que "las relaciones París-Buenos Aires y Londres-Buenos Aires eran a menudo más estrechas que las que unían a las dos

momento). Realizó aportes teóricos como "la etapa pregenital" y las técnicas de juego. Para mayor información sobre la vida de Aberastury consultar: Balán (1991), Borinsky (2009), Winkler Muller y Wolff Reyes (2005). capitales europeas entre sí", de manera que las ideas kleinianas entre Londres y París eran trianguladas a través de Buenos Aires. De hecho, la producción psicoanalítica argentina que, a pesar de ser sumamente original, se expresaba en un lenguaje kleiniano, fue mucho mejor recibida en Francia que la obra de Klein. Esta triangulación fue facilitada por el viaje a Europa en 1951 de Arminda y Enrique Pichon-Rivière, en el que Aberastury obtuvo los derechos de traducción de la obra de Klein y se supervisó en Londres con ella (Dagfal, 2009, p. 138). La posición de Aberastury respecto al intercambio epistolar, las traducciones y, fundamentalmente, la aplicación de estos conocimientos al psicoanálisis de niños (que siguen bastante fielmente a Klein), dan motivos de sobra para que ocupe el lugar central como "receptora" de dicha obra, siempre y cuando se tenga en cuenta que era solo la figura visible de un proceso de implantación que se daba simultáneamente en distintos profesionales del campo disciplinar.

Cabe mencionar que algunas investigaciones desde la perspectiva sociológica y de género se ocuparon de indagar la asociación entre mujeres, psicoanálisis y niños (Balán, 1991; Borinsky, 2009) y otras líneas de investigación se centraron en la relación entre pedagogía y psicoanálisis en la obra de Aberastury (Carli, 2009). Ahora sí, nos detendremos en el caso clínico.

\section{"La inclusión de los padres en el cuadro de la situación analítica"}

A partir de aquí, el trabajo se focalizará en el aspecto de la incorporación de los padres en el psicoanálisis de niños propuesto por esta autora, partiendo del supuesto del carácter problemático que tiene la familia para el análisis infantil.

En 1957, Arminda Aberastury publica en la Revista de Psicoanálisis: "La inclusión de los padres en el cuadro de la situación analítica y el manejo de esta situación a través de la interpretación”’3. Se

3 Este caso fue trabajado anteriormente en: Macchioli y Chayo, «Que faire des parents lors de la psychanalyse d'enfants? Mise au point à partir de l'œuvre d'Arminda Aberastury en Argentine 
"LA INCLUSIÓN DE LOS PADRES EN EL CUADRO DE LA SITUACIÓN ANALÍTICA".

FAMILIA, DISCIPLINAS PSI Y VALORES EN LA ARgENTINA DE LOS CINCUENTA

plantean aquí básicamente los problemas técnicos que la autora encontró, cuando se le presentó el siguiente caso:

H. fue traído a la consulta por padecer de una anorexia seria desde el día en que él mismo, sus familiares y la sociedad lo señalaron como asesino. En los diarios de fines del 55 apareció una nota policial en la que se relataba que un niño de cuatro años había dado muerte a su primo de meses golpeándole la cabeza con un frasco. Señalaban también que cuando encontraron a la víctima, ésta yacía en el suelo ensangrentada, la boca tapada con algodón. (Aberastury, 1957, p. 138)

Luego de que la policía y el juez descartaron trastornos orgánicos a través de un electroencefalograma y otros estudios no especificados, el médico del Hospital de Niños de Buenos Aires "lo envió para ser psicoanalizado" (Aberastury, 1957, p. 138). En el artículo de Aberastury, puede reconstruirse que hubo una o dos entrevistas previas con los padres, y posteriormente por lo menos dos entrevistas con el niño: en la primera estuvo con la madre y en la segunda con el padre, ya que el niño después de ese dramático acontecimiento no quería separarse de sus progenitores ni estos dejarlo solo con nadie, situación que se trasladó a las sesiones.

Aquí pueden señalarse dos vías para estudiar este proceso: 1) la reconstrucción del caso que la analista hizo para sí y 2) el material de las sesiones e interpretaciones que se despliegan en el análisis infantil. Acerca del primero, varios artículos incluidos en la Revista de Psicoanálisis de la APA solían publicar, luego del escrito del autor, las discusiones posteriores que realizaban los miembros de la institución sobre el caso clínico. Lo interesante aquí, es que Aberastury reconstruye el caso al final de la discusión del trabajo en la APA y para ello se basa en el relato de los padres y el material del niño. La psicoanalista concluye que el primo (bebé fallecido)

dans les années cinquante. » [¿Qué hacer con los padres en el psicoanálisis de niños? Puntualizaciones desde la obra de Arminda Aberastury en la Argentina durante la década de 1950]. Trabajo monográfico inédito. se cayó del coche al suelo, cuando el pequeño quiso sacarlo (el cochecito era del niño y se lo habían prestado al primo al nacer) y para no oírle los gritos le tapó la cara con algodón y cuando vio que eso no era eficaz lo golpeó. El padre aseguraba que su hijo había matado al primo "a puertas cerradas", pero en cambio, a medida que se desarrollaba la entrevista, Aberastury no tenía ningún elemento real para afirmar eso, porque el crimen no había pasado estando el padre en la casa del bebé y la madre contó que ella se había entretenido poniéndose una ropas junto a sus amigas y vecinas, mientras su hijo quedó solo con el primo. En un momento determinado, el hijo apareció muy angustiado y le dijo "mamá, vamos", momento en que la madre se dio cuenta de qué algo había sucedido. Para Aberastury, finalmente, "la verdad" era que la madre había dejado al primo en manos del hijo y el padre había "sugerido" la posibilidad del crimen. La reacción del padre respondería, para la autora, a que cuando este era pequeño había planeado en su inconsciencia a "puertas cerradas" el asesinato de su hermano, justamente el padre del sobrino muerto (Aberastury, 1957, p. 146) ${ }^{4}$.

Sobre la segunda vía, al material de las sesiones e interpretaciones en el encuadre analítico infantil, Aberastury le dedica prácticamente todo el artículo. Inaugura el escrito con las dificultades técnicas que se presentan cuando numerosas veces durante el análisis de niños pequeños los padres permanecen durante las entrevistas. Allí surge el problema técnico respecto a cómo manejarse con ellos, y los siguientes interrogantes que se plantea la analista, que ayudan a explicitar algunos problemas de la época: ¿Podía y debía interpretarse delante de los

4 Cinco años después, Aberastury escribirá el primer libro de psicoanálisis de niños en la Argentina: Teoría y técnica del psicoanálisis de niños (1962). Allí, los padres alcanzarán una posición en la técnica del análisis infantil, planteando en la "técnica actual" dos apartados de cuatro al trabajo con los padres sobre la entrevista inicial y las posteriores. Asimismo, con respecto a la psicoprofilaxis de la neurosis infantil, describirá su trabajo con grupos de orientación a madres. No obstante, continuará sosteniendo que conviene suprimir casi totalmente las entrevistas con los padres, excepto cuando manifiesten la necesidad de un encuentro donde podría ser "perturbador" negarla, retomando y reiterando de esta manera, como en 1957, que en la unidad hijo-padres la interpretación debe ir dirigida exclusivamente "al paciente". En definitiva, este problema figura una y otra vez en los textos de este período acerca de quién es el paciente. 
padres aun cuando las interpretaciones fuesen muy dramáticas para ellos?, iSe corría el riesgo de la interrupción del tratamiento si las interpretaciones no podían ser elaboradas por los padres?, ¿Cómo debía manejarse la interpretación en estos casos: teniendo en cuenta la ansiedad de los padres o la necesidad del niño?, ¿Podía incluso llegar a ser valiosa la presencia de los padres? (Aberastury, 1957, p. 137). A lo que Aberastury responde que no solo es posible sino necesario interpretar en dichas circunstancias, pero siempre teniendo en cuenta a la pareja madrehijo o padre-hijo como una sola persona, que para ella es "el paciente".

En el caso clínico puede observarse cómo trabajó algunas de estas cuestiones. En las entrevistas previas con los padres de $\mathrm{H}$., el padre señaló que "el crimen había sido planeado previamente y realizado a puertas cerradas en ausencia de los padres del bebé. Estas dos afirmaciones resultaron ser una fantasía del padre" (Aberastury, 1957, p. 138). La madre, al final de estas entrevistas, reveló que "el cochecito en que estaba acostado el bebé había sido de H. (...). El mismo día del crimen al verlo en casa de su primo lloró y protestó porque lo quería para sî" (Aberastury, 1957, p. 138). El padre, recién en ese momento, recordó que había recomendado a su esposa no dejar solo a $\mathrm{H}$. con el primo "porque lo miraba de un modo raro". Para Aberastury "el padre era el portavoz del niño", reflejado en frases tales como "yo era como él, muy diablo, pero nunca maté a nadie".

A lo largo de ese artículo, pueden ubicarse ciertos núcleos que presentan contradicciones. Por una parte, sostendrá que la interpretación dirigida al niño actuará por igual en el padre, ya que ambos poseen el mismo conflicto, a pesar de que haya sido el hijo el que ejecutó lo que el padre fantaseó en su infancia. Afirma que "es necesario enmarcar la situación analítica en la relación con el niño (...) aún cuando los padres estén incluidos en la sesión" (Aberastury, 1957, p. 142).

Otra dificultad se centra en discutir la validez y el uso del relato de los padres. En este punto la autora ofrece opiniones contrapuestas. Por un lado, le era imposible desentenderse del relato de los padres, ya que el caso especial de un "niño crimi- noso" hace indispensable para la interpretación "el conocimiento del hecho real: H. había matado". Por otro lado, el propio material del niño le permitió luego "interpretar las circunstancias que rodearon el hecho y lo desencadenaron sin tener en cuenta lo narrado por los padres" (Aberastury, 1957, p. 138). Más allá del caso en particular, Aberastury manifiesta que cuando los padres refieren el hecho, se expresa la posición afectiva de ellos frente al suceso, correspondiente a las fantasías de cada uno de ellos. Al mismo tiempo, por momentos ubica al padre como "portavoz" del niño, aunque el uso que hace de este concepto se asemeja más a la identificación que al concepto de "portavoz" de Pichon-Rivière5.

En una nota al pie menciona que la primera sesión podría ser la única en la que el "grupo familiar" se vería tan comprometido en la situación, y en la que los móviles inconscientes del niño puedan observarse más claramente, aunque no vuelve a referirse a la familia. Por otra parte, cuando se refiere a la situación transferencial, menciona que su "rol" como analista será hacerse cargo del crimen, liberándolo al niño de él, que le permite una "libre relación con la madre no contaminada con el odio que le permitiese comer nuevamente de ella" (Aberastury, 1957, p. 141), al tiempo que analiza qué rol le adjudicaron los padres a ella. Según Aberastury, la madre le atribuía a ella el rol de discriminar entre lo bueno y lo malo para su hijo, que generó que la madre se sintiera acusada frente a una interpretación de la psicoanalista, al dejar solo al hijo con el primo haciendo posible el crimen. En cambio el padre se identificaba con la psicoanalista y el hijo, mientras que discriminaba para la analista los motivos del crimen.

Por último, se pesquisa a lo largo del artículo ciertos aspectos que no pertenecen enteramente al marco analítico, aunque Aberastury luego los incluya en el encuadre. Primero, uno de los hijos de Arminda pregunta dónde está su mamá, mientras la psicoanalista estaba atendiendo a H. y su madre, lo

5 Para Pichon, el "portavoz" es aquel miembro que denuncia lo que acontece grupalmente, explicita las fantasías, ansiedades y necesidades del grupo. Este rol conjuga la verticalidad -historia personal- con la horizontalidad -el aquí y ahora del grupo(Pichon-Rivière, 1971, p. 320). 
"LA INCLUSIÓN DE LOS PADRES EN EL CUADRO DE LA SITUACIÓN ANALÍTICA".

FAMILIA, DISCIPLINAS PSI Y VALORES EN LA ARGENTINA DE LOS CINCUENTA

que generó en $\mathrm{H}$. una crisis de ansiedad y se abraza a su madre. Cabe recordar que Arminda tenía su consultorio en su casa. Segundo, cuando la psicoanalista interpreta los celos y el miedo a la separación de H. con su madre, recordando la situación en que el niño luego de la escena del primo se queda solo "con toda la rabia", la madre interrumpe violentamente sosteniendo "iSeñora!, mi marido ordenó que no hay que hablarle más del asunto" (Aberastury, 1957, p. 140). Tercero, el padre hace alusión a un artículo de La Razón, en la que se menciona "eso del inconsciente... de los chicos" (Aberastury, 1957, p. 141), mientras que Aberastury al inicio del artículo señala que este fue un caso público que figuraba en la sección policial de los diarios.

Como solía hacerse en la APA, el trabajo se discutió y las intervenciones de los analistas se centraron en el trabajo con grupos y familias. Dichas intervenciones permiten poner de relieve qué interrogantes y respuestas se ensayaban alrededor de la familia, aunque estas todavía eran escasas en comparación con las referencias sobre las prácticas con niños y grupos.

\section{Algunas reflexiones sobre psicoanálisis de niños, familia, disciplinas psi y valores}

A partir del caso expuesto, pueden indagarse los siguientes niveles en el material clínico: los valores asociados a la salud-enfermedad en juego, a las cuestiones de género, a las representaciones de grupo, familia, padres, niños y paciente.

Sobre el primero, el criterio de normalidad que puede inferirse en este caso supondría en gran medida lo opuesto a lo que allí se manifiesta: niños que se comportan más moderadamente, que frente a los celos o la rabia mantienen disputas que, en el mejor de los casos, entre los mismos niños pueden manejar y si no los padres pueden controlar, niños que se alimentan adecuadamente y que a los cuatro años pueden mantener cierta autonomía de los progenitores. Justamente en el caso, el niño no puede quedarse solo en la sesión, situación que obliga a la analista a incluir a los padres.

La patología en este caso clínico adquiere dos aspectos en el motivo de consulta: el "crimen" y la anorexia. Por la segunda situación, lo derivan rápidamente a Pichon-Rivière; por la primera, luego de exámenes clínicos a cargo del pediatra, se lo derivan a Aberastury. En el artículo, el desplazamiento que traza la psicoanalista es del crimen al accidente. Ya no es que $\mathrm{H}$. quería matar al primo actuando las fantasías homicidas de su padre con su tío cuando eran pequeños, sino los celos y la rabia que $\mathrm{H}$. tenía porque su primo usaba su cochecito. Este desplazamiento desliza la responsabilidad del niño hacia los padres. Al final del artículo, Aberastury insinúa la culpabilidad de la madre al dejar solo a $\mathrm{H}$. con el primo, así como las sugerencias del padre a partir de sus fantasías inconscientes sobre la posibilidad del crimen cuando era pequeño en relación con su hermano. En suma, la mirada sobre lo patológico predomina permanentemente en el texto aunque se desliza la responsabilidad del niño a los adultos y de algo intencional (el crimen) a algo accidental.

Segundo, las cuestiones asociadas al género se manifiestan sobre todo en las mujeres. La madre que probándose ropas con las amigas y vecinas deja solo al niño, pero a la vez es la madre la que se ocupa del niño ya que el padre no está en la escena del "crímen", aunque su palabra nomina la situación como tal cuando sostiene que su hijo "planeó" el crimen y lo hizo "a puertas cerradas". Asimismo, cuando la madre de H. le dice a la psicoanalista "iSeñora!, mi marido ordenó que no hay que hablarle más del asunto", la frase mantiene el esquema patriarcal del marido por sobre la mujer (aunque esto lo sostenga la mujer y lo ponga en palabras del marido). Por otra parte, hallamos a una Aberastury analistamujer-madre que atiende en su casa y que mientras interpreta a su paciente escucha el llamado de su hijo tras la puerta del consultorio, situación con la que debe lidiar en la sesión, explicitándolo en una nota al pie del artículo: tener que dejar de lado "las reacciones que despertó en mí la inclusión de mi hijo en la sesión". En suma, dos mujeres a cargo de sus hijos, tarea asignada socialmente a la mujer en la época (Cosse, 2010; Nari, 2004).

Respecto a las distinciones entre grupo, familia, padres, niños, paciente, solo nos detendremos en los aspectos que se presentan en este artículo. Las contradicciones aquí son varias, dando cuenta de 
la dificultad de recortar el objeto de intervención adecuadamente. Aberastury sostiene que la pareja madre-hijo o padre-hijo operan a nivel técnico como una sola persona, en tanto la interpretación actuará en distintos niveles, ya que "el paciente" incluye al niño, pero también al padre o a la madre. Más adelante sostiene que la interpretación actúa por igual en el padre y el niño por poseer el mismo conflicto. En suma, el "paciente" para la psicoanalista es quien esté en sesión: el niño, la pareja madre-hijo o la pareja padre-hijo, a quienes atribuye las mismas capacidades de elaboración frente a la interpretación.

Pocos años después, la misma Aberastury sostenía que "la validez de los datos suministrados por los padres es muy relativa y podremos saber más a través del niño mismo" (Aberastury, 1962, p. 144). Según Carli, esta postura conduce a la psicoanalista a "desestimar la realización de entrevistas a padres y a dar prioridad al trabajo directo con el niño propiciando un primer descentramiento de los padres y postulación del niño como interlocutor directo del analista cuestión que implica una cambio respecto de la técnica original y una primera apuesta a escuchar la versión del niño". De tal modo que "se pone en juego una confrontación entre la versión conyugal y la versión infantil, tomando el analista partido por esta última. El niño emergía como portador de una verdad que el analista debía decodificar e interpretar, un analista que debía tener “interés por la investigación”" (Carli, 2009, p. 18). No obstante, este caso clínico plantea una paradoja a la psicoanalista: una vez que "usa" el relato de los padres, no puede quedar por fuera de esta versión de los hechos, que además Aberastury considera como "el hecho real" al reconstruir lo sucedido. $\mathrm{Al}$ utilizar en las interpretaciones dicho material, automáticamente le está otorgando validez. Por lo tanto, sostener que solo se basará en el material del niño sin tener en cuenta lo narrado por los padres, parecería ser más bien una formación de compromiso, entre el modelo teórico kleiniano, las analogías con el psicoanálisis de adultos y las prácticas con niños y sus familiares. Esto supone a la vez, que hay un relato "verdadero" que corresponde al mundo interno y las fantasías inconscientes, en este caso del niño, pero no se sabe como compatibilizar esto con el psiquismo de los padres, porque permanentemente se piensa epistémicamente en un sujeto y no en un conjunto de personas que producen otros fenómenos diversos a la lógica individual.

Así, el "grupo familiar" solo figura una vez en el artículo en una nota al pie referido al fuerte compromiso que este grupo familiar presentaba frente a la situación del "crimen". Y aunque Aberastury se refiere a ideas de corte pichoneano como "grupo familiar", "portavoz", "asunción y adjudicación de roles", "emergente" y "grupo familiar", trasmitidas por Pichon un año antes en un curso de la APA, luego editadas en Teoría del vínculo (1980), son ideas casi ausentes en los desarrollos de Aberastury ${ }^{6}$. Este hecho incluso llama la atención cuando, al discutirse el trabajo en el seno de la APA, las intervenciones se centraron en el incipiente trabajo con grupos y familias que ya estaba cobrando forma por fuera y por dentro de la asociación con figuras como E. Pichon-Rivière, R. Usandivaras, E. Rolla o E. Rodrigué, entre otros.

Por otra parte, según Carli, la antítesis entre autoridad y libertad que operó como una antinomia clásica de la historia de la pedagogía, encuentra en el psicoanálisis una nueva inflexión que puede pesquisarse en la obra de Aberastury. Si en los cuarenta el psicoanálisis intervenía refutando los efectos de una escuela y una familia de corte tradicional, en los sesenta debía intervenir en un ámbito en el que las transformaciones familiares, los procesos de renovación pedagógica en las escuelas y las innovaciones socioculturales de la segunda posguerra favorecían modalidades más permisivas de crianza y educa-

6 Sobre el vínculo en la obra de Pichon-Rivière pueden precisarse algunos aspectos: primero, desde la perspectiva de una historia conceptual, el vínculo puede rastrearse desde la década de 1940 a partir de cierto proto-vínculo inaugurado en la relación del feto con la madre en el transcurso del embarazo; segundo, cierto modo de recepción en la obra del psiquiatra argentino superpone en el concepto de vínculo la relación de objeto freudo-kleiniana, la teoría de los roles de G. Mead y la dinámica de grupos de K. Lewin, operación que hace del término una zona heterogénea de encuentro entre diversas zonas geográficas y tradiciones disciplinares; y finalmente, se debe atender a los modos en que el vínculo se constituyó como surco privilegiado de lo que generalmente se planteó como una tercera y última etapa en la obra de PichonRivière asociada a la psicología social (Macchioli, en prensa). 
"LA INCLUSIÓN DE LOS PADRES EN EL CUADRO DE LA SITUACIÓN ANALÍTICA".

FAMILIA, DISCIPLINAS PSI Y VALORES EN LA ARgENTINA DE LOS CINCUENTA

ción. No obstante, en el caso de la psicoanalista argentina, no hay que perder de vista el ensamble entre su formación pedagógica en simultáneo a su primer acercamiento al psicoanálisis. En el caso del psicoanálisis de niños en particular, "su verdad sobre el niño borra las contingencias y derroteros de su conformación y en particular sus operaciones de diferenciación con otros discursos (en este caso el pedagógico)" (Carli, 2009, p. 14, 23), así también, como se ha desarrollado hasta aquí, el discurso pediátrico, criminológico y médico, entre otros.

Finalmente, cabe afirmar a partir de este caso, cierta coyuntura psi descripta al inicio del artículo. Por una parte, el caso de H., que cobró estado público a partir de los diarios nacionales de mediados de los cincuenta, es derivado a una psicoanalista de niños, lo que permite suponer que esta especialidad era reconocida y estaba legitimada en el campo disciplinar y jurídico. Este desenlace está en sintonía con los desarrollos científicos alrededor del problema del niño que se inician desde fines del siglo XIX en la Argentina, vinculados a preocupaciones urbanas y sociales. Este caso en particular, si el acento se ubica en la criminalidad infantil, pone de relieve las características mencionadas acerca de las repercusiones en la opinión pública, la intervención del Estado a partir de políticas sociales, la involucración del ámbito familiar privado y los conocimientos e intervenciones profesionales inseparables de las ideas y de las políticas públicas vigentes a mediados de los cincuenta. En el caso de Aberastury, H. primero es examinado por la policía y el juez que excluyeron trastornos orgánicos a partir de estudios médicos, para que luego el médico interviniente recomendara un psicoanálisis infantil que, en la práctica, debió inexorablemente involucrar a los padres.

Por otra parte, la derivación a Aberastury por la situación "criminal" y a Pichon-Riviére por anorexia permite entrever al interior de la asociación psicoanalítica la diversidad en los modos de intervención de un mismo caso: por una parte, Aberastury para ese entonces sería ubicada como representante de la ortodoxia kleiniana dentro de la APA, mientras que Pichon iría tomando cada vez mayor distancia de la institución para emplazarse en diversos ámbitos que darían paso a lo que posteriormente sus discípulos denominarían "psicología social" protagonizada por los grupos?

El caso de Aberastury, publicado en 1957, se instala en una agenda psi muy nutrida de cara a la conformación del movimiento de la Salud Mental no solo en la Argentina sino también en Latinoamérica, que dio lugar al establecimiento de diversas prácticas como la psicoterapia de grupo, la Escuela para Padres y, poco más tarde, a las intervenciones comunitarias, institucionales y familiares, entre muchos otros dispositivos clínicos. El escenario presentado no hace más que continuar un proceso de psicologización creciente que se extendió del adulto al niño para incluir poco después al grupo y la familia.

\section{Referencias}

Aberastury de Pichon Rivière, A. (1957). La inclusión de los padres en el cuadro de la situación analítica y el manejo de esta situación a través de la interpretación. Revista de Psicoanálisis, 14(1-2), 137-146.

Aberastury, A. (1962). Teoría y técnica del psicoanálisis de niños. Buenos Aires: Paidós.

Balán, J. (1991). Cuéntame tu vida. Una biografía colectiva del psicoanálisis argentino. Buenos Aires: Planeta.

Bardassi, T. (2009). Los orígenes de psicoanálisis de niños en Brasil: entre la educación y la medicina. Psicologia em Estudo, 14(3), 423-432.

Benito, E., \& García, L. (2010). Psicología en sociedad. Psiencia, 2(2), 60-69.

Borinsky, M. (2009). Historia de la práctica terapéutica con niños, psicología y cultura (1940-1970). La construcción de la infancia como objeto de intervención psicológica. (Disertación doctoral no publicada). Facultad de Psicología, Universidad de Buenos Aires.

7 Cabe aclarar que para Pichon-Rivière toda psicología es social, por tanto, sería redundante plantear una "psicología social". A diferencia del sujeto freudiano, el sujeto para Pichon emerge en el interior de una red de vínculos, donde las experiencias con otros darán lugar a las vivencias de satisfacción y de frustración, en lugar de originarse en el instinto sexual como lo propone la teoría psicoanalítica (Macchioli, en prensa; Pichon-Rivière, 1971). 
Carli, S. (2009). La infancia como verdad. Las tensiones entre psicoanálisis y educación en la obra de Arminda Aberastury. Temas de Historia de la Psiquiatría Argentina, 29, 13-26. Recuperado de http://www. polemos.com.ar/docs/temas/temas29.pdf

Carpintero, E., \& Vainer, A. (2004). Las huellas de la memoria (Vols. 1-2). Buenos Aires: Topía.

Cosse, I. (2010). Pareja, sexualidad y familia en los años sesenta. Buenos Aires: Siglo XXI.

Dafermos, M., Marvakis, A., Mentinis, M., Painter, D., \& Triliva, S. (Eds.). (2013). Critical psychology in a changing world: Bilding bridges and expanding the dialogue. Annual Review of Critical Psychology, 10, 1-33.

Dagfal, A. (2009). Entre París y Buenos Aires. Buenos Aires: Paidós.

Dorlin, E. (2009). Sexo, género y sexualidades. Buenos Aires: Nueva Visión.

Dotti, A., Blanco, A., Plotkin, M., García, L. I., \& Vezzetti, H. (2008). Encuesta sobre el concepto de recepción. Políticas de la Memoria, 8-9, 98-109.

Geissmann, C., \& Geissmann, P. (2002). Historia del psicoanálisis infantil. Madrid: Síntesis.

Macchioli, F. (2010). Los inicios de la terapia familiar en la Argentina. Implantación, configuración y desarrollo de un nuevo campo disciplinar. 1960-1979. (Disertación doctoral no publicada). Facultad de Medicina, Universidad de Buenos Aires.

Macchioli, F. (2012). Terapia familiar y teoría de la comunicación humana. Acta Psiquiátrica y Psicológica de América Latina, 58(3), 201-209.

Macchioli, F. (2013). Familia y salud mental en la Argentina de 1957. En Salud Mental: Interdisciplina e inclusión social como ejes de intervención (Vols. 2, pp. 486-489). Buenos Aires: Asociación Argentina de Salud Mental.

Macchioli, F. (en prensa). Enrique Pichon-Rivière y los saberes psi en la Argentina. Reflexiones sobre la conformación del vínculo entre la psiquiatría, el psicoanálisis y la psicología. Psychologia Latina, 4.

Nari, M. (2004). Políticas de maternidad y maternalismo político. Buenos Aires: Biblos.

Oliveira, C. L. de (2002). Os primeiros tempos da psicanálise no Brasil e as teses pansexualistas na educação. Ágora, 5(1), 133-154. Recuperado de http:// www.fundamentalpsychopathology.org/uploads/ files/ revistas/volume05/n3/a_historiografia_sobre_o_movimento_psicanalitico_no_brasil.pdf

Pichon-Rivière, E. (1971). Del psicoanálisis a la psicología social (Vol. 2). Buenos Aires: Galerna.

Pichon-Rivière, E. (1980). Teoría del vínculo. Buenos Aires: Nueva Visión.

Plotkin, M. (2003). Freud en las pampas. Buenos Aires: Sudamericana.

Prilleltensky, I. (1997). Values, assumptions, and practices: Assessing the moral implications of psychological discourse and action. American Psychologist, 52, 517-535.

Puget, J. (2011). Comunicación personal, 26-11-11.

Pulido Martínez, H. (2007). Produciendo trabajadores modernos: conocimiento psicológico y el mundo del trabajo en el sur. Universitas Psychologica, 6(1), 27-37.

Talak, A. M. (2009). Historia y epistemología de la psicología: razones de un encuentro necesario. En D. Letzen \& P. Lodeyro (Eds.), Epistemología e historia de la ciencia (Vol. 15, pp. 477-482). Córdoba: Universidad Nacional de Córdoba.

Talak, A. M. (en prensa). El desarrollo psicológico entre la naturaleza, la cultura y la política (1900-1920). En L. García, F. Macchioli \& A. M. Talak (Eds.), Psicología, niño y familia en la Argentina (1900. 1970). Perspectivas históricas y cruces disciplinares. Buenos Aires: Biblos.

Tarcus, H. (2007). Marx en la Argentina. Sus primeros lectores obreros, intelectuales y científicos. Buenos Aires: Siglo XXI.

Torrado, S. (2004). La herencia del ajuste. Buenos Aires: Capital Intelectual.

Vera-Villarroel, P., López-López, W., Lillo, S., \& Silva, L. M. (2011). La producción científica en psicología latinoamericana: un análisis de la investigación por países. Revista Latinoamericana de Psicología, 43(1), 95-104.

Vezzetti, H. (1985). La locura en la Argentina. Buenos Aires: Paidós.

Vezzetti, H. (1996a). Aventuras de Freud en el país de los argentinos. De José Ingenieros a Enrique PichonRivière. Buenos Aires: Paidós.

Vezzetti, H. (1996b). Freud en Buenos Aires. Bernal: Universidad de Quilmes. 
"LA INCLUSIÓN DE LOS PADRES EN EL CUADRO DE LA SITUACIÓN ANALÍTICA".

FAMILIA, DISCIPLINAS PSI Y VALORES EN LA ARGENTINA DE LOS CINCUENTA

Vezzetti, H. (2007a). Historias de la psicología: problemas, funciones, objetivos. Revista de Historia de la Psicología, 28(1), 147-166.

Vezzetti, H. (2007b). El psicoanálisis en el siglo. Punto de Vista, 88 .
Wainerman, C. (2005). La vida cotidiana en las nuevas familias. Buenos Aires: Lumiere.

Winkler Muller, M. I., \& Wolff Reyes, X. (2005). "El Buenos Aires kleiniano". Vida y obra de Arminda Aberastury (1910-1971). Acheronta, 22. Disponible en www.acheronta.org 
